\title{
A model for Neutrino Masses and Dark Matter with the Discrete Gauge Symmetry
}

\author{
We-Fu Chang*t and Chi-Fong Wong \\ Department of Physics, National Tsing Hua University, HsinChu 300, Taiwan
}

\begin{abstract}
A simple renormalizable $U(1)$ gauge model is constructed to explain the smallness of the active neutrino masses and provide the stable cold dark matter candidate simultaneously. The local $U(1)$ symmetry is assumed to be spontaneously broken by a scalar field around the TeV scale. The active neutrino masses are then generated at one-loop level. This model contains several cold dark matter candidates whose stability is guaranteed by a residual discrete gauge $Z_{2}$ symmetry a la the Krauss-Wilczek mechanism. Unlike the other dark matter models, no further global discrete or continuous symmetry is introduced. Moreover, the masses of all fermionic degrees of freedom beyond the Standard Model are closely related to the scale of spontaneous breaking of $U(1)$ thus they could be probed at or below the TeV scale. The possible cosmological and phenomenological consequences are briefly discussed.
\end{abstract}

*Electronic address: wfchang@phys.nthu.edu.tw 


\section{INTRODUCTION}

It is now widely accepted that at least two kinds of neutrinos are massive[1]. The pressing questions we are facing now: (1) how many extra degrees of freedom beyond the Standard Model (SM) are responsible for generating the neutrino masses, and (2) are they fermionic or bosonic? On the phenomenological side, it is practical to ask whether we are able to test the neutrino mass generation mechanism directly. If the new degrees of freedom decouple at a rather high energy scale, unfortunately this happens for some of the cases, we are left with only one effective dim-5 operator $(L \Phi)^{2}$ at low energy. For example, there is no way to directly test the usual seesaw mechanism[2] with $\sim 10^{14} \mathrm{GeV}$ or higher Majorana neutrinos. And we can only study its indirect consequences, for example, the leptogenesis. It will be interesting, although unnecessary, to have a neutrino mass generation mechanism and at the same time the responsible new degrees of freedom are accessible within human's reach. It was first pointed out by Zee and Babu[3] that the active neutrino masses could be generated radiatively. In that case, due to the loop suppression factors, the active neutrino masses are naturally small compared to other SM charged fermions. Although the masses of the new degrees of freedom are totally arbitrary, they are more likely to be probed experimentally in some parameter space. Therefore, we aim for a model in which the neutrino masses are radiatively generated ${ }^{1}$. Moreover, we hope to have a unified mechanism such that the mass scale of the new degrees of freedom is not totally arbitrary.

On the other hand, there are several ways to infer the dark matter abundance $\Omega_{D M}$ in the Universe[5]. The existence of dark matter with $\Omega_{D M} h^{2} \sim 0.11$, where $h$ is the Hubble constant in units of $100 \mathrm{~km} /(\mathrm{s} . \mathrm{Mpc})$, requires physics beyond the SM. It will be even more interesting if one of the new fields for radiatively generating neutrino mass serves as the dark matter candidate. In fact, several interesting models have been proposed to connect the origin of neutrino masses to the existence of the cold dark matter [6, 7]. And, a viable dark matter candidate must be as long-lived as the Universe. It is common for people to impose global discrete or continuous symmetry to stabilize the dark matter candidate from decaying ( [7] is an exception, but it failed to come up with a theory which is renormalizable and free of anomalies. ) However, the origin of these global discrete or continuous symmetries is not

1 For the follow ups and other proposals for generating neutrino mass, readers may want to consult a recent review [4]. 
explained. Moreover, quantum gravity effects do not respect global symmetries [8]. One elegant remedy is to use the discrete symmetry originated from a spontaneously broken gauged symmetry, known as the Krauss-Wilczek mechanism(KWM) [8]. For a recent implementation of KWM to stabilize the dark matter candidate see [9] and references therein.

In this paper, a $U(1)_{\nu}$ gauge symmetry is introduced on top of the SM interactions and it is responsible for all three features mentioned above: neutrino masses generation, testable new degrees of freedom, and the existence of stable dark matter. The $U(1)_{\nu}$ is spontaneously broken by a SM singlet scalar which carries two units of $U(1)_{\nu}$ charge. A gauged discrete $Z_{\nu 2}$ symmetry can remain after the spontaneous symmetry breaking (SSB) of $U(1)_{\nu}$ and the $Z_{\nu 2}$-odd cold dark matter candidate can be stable. While each ingredient is not new, to our best knowledge, ours is the first successful model which conjoins all.

\section{MODEL SETUP}

We present a minimal model which makes use of the KWM and radiatively generates the neutrino masses at the lowest possible mass dimension. In addition to the SM fermions and Higgs doublet $\Phi$, this model consists of two extra pairs of chiral fermions $N_{R_{1,2}}$ and $n_{L_{1,2}}$, one extra Higgs doublet $\eta$, and two complex scalars $S$ and $\sigma$. Their quantum numbers are summarized in Table I. The $U(1)_{\nu}$ charge assignment forbids the $N_{R a}$ and $n_{L a}$ to have Majorana masses. Note that we need at least two pairs of $N_{R}$ and $n_{L}$ to accommodate the observed neutrino data. Introducing vector-like fermions is needed for cancelling the anomalies. In this model, the $U(1)_{\nu}$ invariant Yukawa couplings and the Dirac mass term

\begin{tabular}{|c|ccc|cc|cc|cccc|}
\hline & $Q_{L i}$ & $u_{R i}$ & $d_{R i}$ & $L_{i}$ & $e_{R i}$ & $N_{R a}$ & $n_{L b}$ & $\Phi$ & $\eta$ & $\sigma$ & $S$ \\
\hline$S U(2)_{L}$ & 2 & 1 & 1 & 2 & 1 & 1 & 1 & 2 & 2 & 1 & 1 \\
$U(1)_{Y}$ & $\frac{1}{6}$ & $\frac{2}{3}$ & $-\frac{1}{3}$ & $-\frac{1}{2}$ & -1 & 0 & 0 & $\frac{1}{2}$ & $\frac{1}{2}$ & 0 & 0 \\
\hline$U(1)_{\nu}$ & 0 & 0 & 0 & 0 & 0 & -1 & -1 & 0 & -1 & -1 & 2 \\
\hline$Z_{2 \nu}$ & + & + & + & + & + & - & - & + & - & - & (N.A.) \\
\hline
\end{tabular}

TABLE I: Charge assignment and the remaining discrete $Z_{2 \nu}$ parity for the fields, where $i=1,2,3$, $a, b=1,2$, and $Q_{L}, u_{R}, d_{R}, L, e_{R}$ are the standard notation for the SM quark and lepton. 
between $N_{R a}$ and $n_{L b}$ are:

$$
\frac{y_{a}^{N}}{2} \overline{N_{a}^{C}} S N_{a}+\frac{y_{a}^{n}}{2} \overline{n_{a}^{C}} S n_{a}+g_{i a} \overline{L_{i}} \tilde{\eta} N_{a}+m_{a b}^{D} \bar{n}_{a} N_{b}+h . c .
$$

If the first two terms were absent ( or $y^{N, n}=0$ ), a global axial $U(1)_{A}$ symmetry which transforms $N_{R} \rightarrow e^{i \theta} N_{R}$ and $n_{L} \rightarrow e^{-i \theta} n_{L}$ will forbid the Dirac mass term. The presence of the first two terms explicitly violates the $U(1)_{A}$ symmetry and thus suggests a natural scale for $m_{a b}^{D} \sim y\langle S\rangle$, although it is perfectly legitimate for $m_{a b}^{D}$ to take ANY other value. In this work, we simply focus on the scenario that $m^{D}$ is around $\mathrm{TeV}$ from the standpoint of being phenomenologically interesting. The Yukawa couplings $y^{N}$ and $y^{n}$ can be taken to be diagonal without losing any generality. The most general renormalizable scalar potential in this model is

$$
\begin{aligned}
V & =\bar{\mu}_{\Phi}^{2}|\Phi|^{2}+\bar{\mu}_{\eta}^{2}|\eta|^{2}+\bar{\mu}_{\sigma}^{2}|\sigma|^{2}+\bar{\mu}_{S}^{2}|S|^{2} \\
& +\bar{\lambda}_{1}|\Phi|^{4}+\bar{\lambda}_{2}|\eta|^{4}+\bar{\lambda}_{3}|\sigma|^{4}+\lambda_{4}|S|^{4} \\
& +\bar{\lambda}_{5}|\Phi|^{2}|\eta|^{2}+\lambda_{6}\left|\Phi^{\dagger} \eta\right|^{2}+\bar{\lambda}_{7}|\Phi|^{2}|\sigma|^{2}+\lambda_{8}|\Phi|^{2}|S|^{2} \\
& +\bar{\lambda}_{9}|\eta|^{2}|\sigma|^{2}+\lambda_{10}|\eta|^{2}|S|^{2}+\lambda_{11}|\sigma|^{2}|S|^{2} \\
& +\kappa\left(\Phi^{\dagger} \eta \sigma S\right)+\mu_{1}(\sigma \sigma S)+\mu_{2}\left(\eta^{\dagger} \Phi \sigma\right)+\text { h.c. }
\end{aligned}
$$

The parameters $\kappa, \mu_{1,2}$ can be taken to be real positive. However, this potential is too complicated for one to obtain any meaningful constraint on the parameters. Instead of going for a full analysis of Eq.(2), we assume that $S$ gets a positive real vacuum expectation value $(\mathrm{VEV}), v_{S} \sim T e V$. We assume that the mass of $S,\left(=\sqrt{-2 \bar{\mu}_{S}^{2}}\right)$, is around $\mathrm{TeV}$ as well, such that $\lambda_{4}=-\bar{\mu}_{S}^{2} / 2 v_{S}^{2} \leq 1$ can be met and the scalar sector is still perturbative. When the energy scale is less than $\Lambda=\sqrt{-2 \bar{\mu}_{S}^{2}} \sim \mathrm{TeV}$, the degrees of freedom of $S$ are integrated out and we work with an effective theory without $S$.

The covariant derivative of $S$ is given by $D_{\mu} S=\left(\partial_{\mu}-i g_{\nu} X_{\mu}\right) S$, where $X_{\mu}$ is the $U(1)_{\nu}$ gauge field, and $g_{\nu}$ the gauge coupling constant which should take a value similar to the $\mathrm{SM}$ ones. After SSB, $S$ is parametrized as $S=\left(v_{S}+S_{R}\right) \exp \left(i \tau_{S} / v_{S}\right)$. The Goldstone field, $\tau_{S}$, can be removed by a gauge transformation $X_{\mu} \rightarrow X_{\mu}-\left(\partial_{\mu} \tau_{S} / g_{\nu} v_{S}\right)$ accompanied by a concomitant redefinition of all other fields, $f$, which carry $U(1)_{\nu}$ charge $Q_{f}: f \rightarrow$ $f \exp \left(-i \tau_{S} Q_{f} / 2 v_{S}\right)$. It is clear that a transformation $\tau_{S} \rightarrow \tau_{S}+2 \pi v_{S}$ leaves the background vacuum configuration invariant. The $Z_{\nu 2}$ symmetry then emerges since the charges of all fields other than $S$ are either $\pm 1\left(Z_{\nu 2}\right.$-odd $)$ or $0\left(Z_{\nu 2}\right.$-even $)$ under the $U(1)_{\nu}$. 
The gauge boson of $U(1)_{\nu}$, dubbed $Z_{\nu}^{\prime}$, thus gets a mass $\sqrt{2} g_{\nu} v_{S} \lesssim \mathrm{TeV}$. The singlet fermions $N_{a}$ and $n_{a}$ now acquire their Majorana masses $\left(y_{a}^{N} v_{S}\right)$ and $\left(y_{a}^{n} v_{S}\right)$ respectively. Four Majorana states $\chi_{1-4}$ can be constructed from $N_{a}$ and $n_{a}$ and their charge conjugate by diagonalizing a $4 \times 4$ mass matrix. Since we do not attempt to fit the neutrino oscillation data in this letter, the explicit form of the mixing is not our concern here. The bottom line is that the mixings between the $n_{L}$ and $N_{R}$ sectors are order one, $\tan 2 \theta_{N n} \sim m^{D} / v_{S}\left(y^{N}-y^{n}\right)$. We denote the lightest(heaviest) mass eigenstate as $\chi_{1}\left(\chi_{4}\right)$.

The active neutrinos receive their Majorana masses via the one-loop diagrams displayed in Fig!1. From the effective operator point of view, the active neutrino masses are attributed
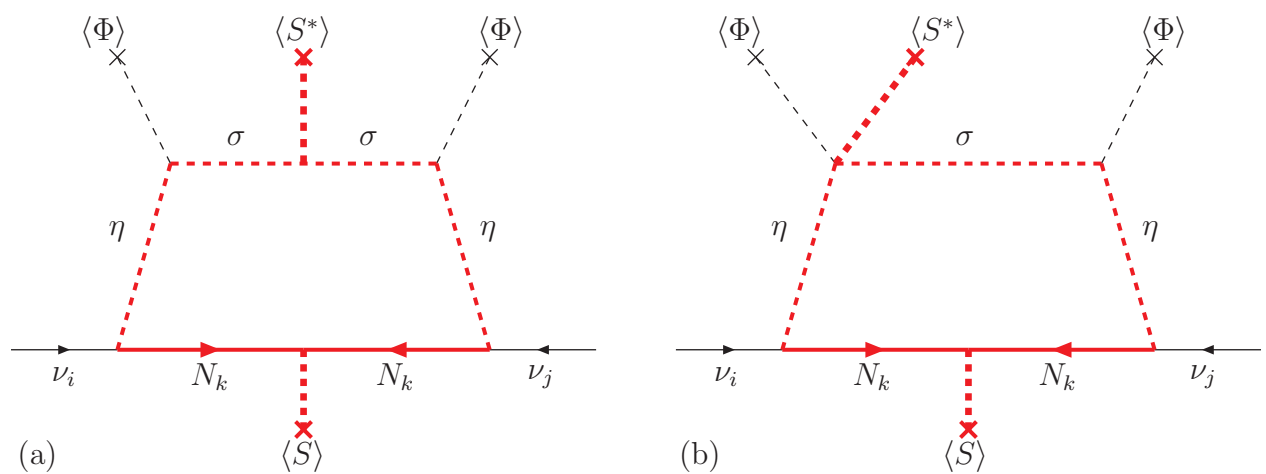

FIG. 1: The 1-loop diagrams which give rise to the active neutrino masses. The mirror image of diagram-(b) which has the $\left\langle S^{*}\right\rangle$ attached to the $\sigma \eta\langle\Phi\rangle$ vertex on the right-hand side is not shown. Those charged under $U(1)_{\nu}$ are the red(thick) lines.

to a dimension-seven operator $(\Phi L)^{2}\left(S^{\dagger} S\right)$. The resulting neutrino mass matrix element $\left(m_{\nu}\right)_{i j}$ from Fig.-1(a) and Fig.-1(b) can be estimated to be

$$
\left(m_{\nu}\right)_{i j}^{(a)} \sim \frac{\mu_{1} \mu_{2}^{2} v_{\Phi}^{2} v_{S}^{2}}{16 \pi^{2} \Lambda^{6}} \sum_{a} y_{a}^{N} g_{i a}^{*} g_{j a}^{*}, \text { and }\left(m_{\nu}\right)_{i j}^{(b)} \sim \frac{\kappa \mu_{2} v_{\Phi}^{2} v_{S}^{2}}{16 \pi^{2} \Lambda^{4}} \sum_{a} y_{a}^{N} g_{i a}^{*} g_{j a}^{*}
$$

respectively, where $\langle\Phi\rangle=v_{\Phi}=174 \mathrm{GeV}$ is the VEV of the SM Higgs. If taking the dimensional couplings $\mu_{1}$ and $\mu_{2}$ to be electroweak $\sim 0.1 \Lambda$, then $m_{\nu}^{(b)} / m_{\nu}^{(a)} \sim\left(\kappa \Lambda^{2} / \mu_{1} \mu_{2}\right) \gg 1$ and Fig.-1(b) gives the dominant contribution to active neutrino masses

$$
\sim 0.01 \times \frac{|g|^{2}}{16 \pi^{2}} \kappa y^{N} \mu_{2}
$$

In Eq.(4), if we take a rather conservative estimation that $\kappa y^{N} \sim 0.1, \mu_{2} \sim 100 \mathrm{GeV}$, and Yukawa coupling $|g| \sim 10^{-4}$ ( roughly 10 times of the Yukawa coupling for the SM electron) the resulting neutrino masses are in the sub-eV range without much fine-tuning. Moreover, 
if $m^{D} \sim y v_{S}$, the Majorana states, $\chi$ 's, are still around but slightly below the $\mathrm{TeV}$ range and could be studied by the man-made machines.

Note that there are two-loop contributions to active neutrino masses if the two external $S$-legs in Fig.-11 are connected. And those two-loop contributions are equivalent to the dimension-five operator $(\Phi L)^{2}$ in low-energy effective theory. With the help of dimensional analysis, their magnitudes can be estimated to be

$$
\left(m_{\nu}\right)_{i j, 2 \text {-loop }}^{(a)} \sim \frac{\mu_{1} \mu_{2}^{2} v_{\Phi}^{2}}{\left(16 \pi^{2}\right)^{2} \Lambda^{4}} \sum_{a} y_{a}^{N} g_{i a}^{*} g_{j a}^{*}, \text { and }\left(m_{\nu}\right)_{i j, 2 \text {-loop }}^{(b)} \sim \frac{\kappa \mu_{2} v_{\Phi}^{2}}{\left(16 \pi^{2}\right)^{2} \Lambda^{2}} \sum_{a} y_{a}^{N} g_{i a}^{*} g_{j a}^{*}
$$

respectively. Again, the connected diagram-1(b) gives dominant two-loop contribution to neutrino masses. In a perturbative theory, the one-loop diagrams are usually much more important than the two-loop diagrams. However, in this model, the one-loop contributions vanish before the SSB of $U(1)_{\nu}$. At that $U(1)_{\nu}$-symmetric stage, the active neutrino masses are controlled by the two-loop diagrams where the cutoff should be replaced by the masses of heavy scalars running in the loop. In some interesting scenarios where $\left(v_{S} / \Lambda\right)^{2} \leq 1 /\left(16 \pi^{2}\right)$, the active neutrino masses are also governed by the two-loop contributions. In those cases, the gauge boson $Z_{\nu}^{\prime}$, the neutral scalar $S_{R}$ (if the theory is still perturbative ), and some of the Majorana states, $\chi$ 's, are all light, $\sim v_{S}$, and make the terrestrial experimental probes more probable. But the following effective theory treatment will not be suitable for a light $S_{R}$ and we leave detailed exploration of this direction for future study.

To simplify the discussion, below $\Lambda$, we assume that $S_{R}$ decouples from the rest and yields an effective potential:

$$
\begin{array}{r}
V_{\text {eff }} \simeq \mu_{\Phi}^{2}|\Phi|^{2}+\mu_{\eta}^{2}|\eta|^{2}+\mu_{\sigma}^{2}|\sigma|^{2}+\lambda_{1}|\Phi|^{4}+\lambda_{2}|\eta|^{4} \\
+\lambda_{3}|\sigma|^{4}+\lambda_{5}|\Phi|^{2}|\eta|^{2}+\lambda_{6}\left|\Phi^{\dagger} \eta\right|^{2}+\lambda_{7}|\Phi|^{2}|\sigma|^{2} \\
+\lambda_{9}|\eta|^{2}|\sigma|^{2}+\kappa v_{S}\left(\Phi^{\dagger} \eta \sigma\right)+\mu_{1} v_{S}(\sigma \sigma)+\mu_{2}\left(\eta^{\dagger} \Phi \sigma\right)+\text { h.c. }
\end{array}
$$

where $\mu_{\Phi}^{2}=\left(\bar{\mu}_{\Phi}^{2}+\lambda_{8} v_{S}^{2}\right), \mu_{\eta}^{2}=\left(\bar{\mu}_{\eta}^{2}+\lambda_{10} v_{S}^{2}\right)$ and $\mu_{\sigma}^{2}=\left(\bar{\mu}_{\sigma}^{2}+\lambda_{11} v_{S}^{2}\right)$. The quartic couplings 
also receive contributions from tree-level $S$ exchange diagrams:

$$
\begin{aligned}
& \lambda_{1}=\bar{\lambda}_{1}+\mathcal{O}\left(\lambda_{8}^{2}\right), \\
& \lambda_{2}=\bar{\lambda}_{2}+\mathcal{O}\left(\lambda_{10}^{2}\right), \\
& \lambda_{3}=\bar{\lambda}_{3}+\mathcal{O}\left(\lambda_{11}^{2}\right)+\mathcal{O}\left(\frac{\lambda_{11} \mu_{1}}{\Lambda}\right)+\mathcal{O}\left(\frac{\mu_{1}^{2}}{\Lambda^{2}}\right), \\
& \lambda_{5}=\bar{\lambda}_{5}+\mathcal{O}\left(\lambda_{8} \lambda_{10}\right), \\
& \lambda_{7}=\bar{\lambda}_{7}+\mathcal{O}\left(\lambda_{8} \lambda_{11}\right)+\mathcal{O}\left(\frac{\lambda_{8} \mu_{1}}{\Lambda}\right), \\
& \lambda_{9}=\bar{\lambda}_{9}+\mathcal{O}\left(\lambda_{10} \lambda_{11}\right)+\mathcal{O}\left(\frac{\lambda_{10} \mu_{1}}{\Lambda}\right) .
\end{aligned}
$$

Since we assume that $\mu_{1} \sim 0.1 \Lambda$ and our model is in the perturbative region as well, these effects and the explicit values of $\lambda$ 's are not important to our current discussion. This potential is bounded from below if all $\lambda$ 's are set to be positive. The $Z_{\nu 2}$ symmetry will be broken if any of $\eta$ or $\sigma$ develop nonzero $\mathrm{VEV}$, but it is straightforward to verify that the true minimum solution that $\langle\Phi\rangle=v_{\phi}=\sqrt{-\mu_{\Phi}^{2} / 2 \lambda_{1}}$ and $\langle\eta\rangle=\langle\sigma\rangle=0$ can be easily accommodated in Eq.(6) . For simplicity, here we do not consider spontaneous CP violation and the nonrenormalizable operators which are irrelevant to the current study.

Both the mixing between $\Phi$ and $\eta$ or $\sigma$ and the mixing between the SM neutrinos and $\chi$ 's are forbidden by the $Z_{2 \nu}$ symmetry. There is only one $Z_{2 \nu}$-even physical scalar field which is identified as the the SM Higgs $h^{0}$, and six ( 2 charged, 2 scalar, and 2 pseudoscalar ) extra $Z_{2 \nu}$-odd physical Higgs. This feature is very different from the other multi-Higgs models. The masses of the $Z_{2 \nu}$-odd Higgs are free but expected to be around sub-electroweak to TeV scale. Due to the $Z_{2 \nu}$ parity, the sterile fermions in this model do NOT mix with the active neutrinos; therefore this model cannot explain the LSND/MiniBoone anomalies [10].

\section{PHENOMENOLOGY}

For the $Z_{2 \nu}$-odd scalar sector, it is easy to work out the mass square $M_{ \pm}^{2}=\mu_{\eta}^{2}+\lambda_{5} v_{\Phi}^{2}$ for

the charged Higgs, and the mass square matrices for the scalar and pseudoscalar bosons are

$$
\begin{aligned}
& M_{\text {odd }}^{s}=\left(\begin{array}{cc}
M_{ \pm}^{2}+\lambda_{6} v_{\Phi}^{2} & \mu_{2} v_{\Phi}+\kappa v_{S} v_{\Phi} \\
\mu_{2} v_{\Phi}+\kappa v_{S} v_{\Phi} & \mu_{\sigma}^{2}+\lambda_{7} v_{\Phi}^{2}+2 \mu_{1} v_{S}
\end{array}\right), \\
& M_{\text {odd }}^{p}=\left(\begin{array}{cc}
M_{ \pm}^{2}+\lambda_{6} v_{\Phi}^{2} & \mu_{2} v_{\Phi}-\kappa v_{S} v_{\Phi} \\
\mu_{2} v_{\Phi}-\kappa v_{S} v_{\Phi} & \mu_{\sigma}^{2}+\lambda_{7} v_{\Phi}^{2}-2 \mu_{1} v_{S}
\end{array}\right),
\end{aligned}
$$


in the basis of $\left\{\operatorname{Re} \eta^{0}, \operatorname{Re} \sigma^{0}\right\}$ and $\left\{\operatorname{Im} \eta^{0}, \operatorname{Im} \sigma^{0}\right\}$ respectively. For the convenience of latter discussion, we denote the mass eigenstates of physical scalar/pseudoscalar as $H_{1,2} / A_{1,2}$, and the subscript $1 / 2$ stands for the lighter/heavier ones. We parametrize the mixing angles $\alpha$ and $\delta$ as $H_{1}=\cos \alpha \operatorname{Re} \eta^{0}+\sin \alpha \operatorname{Re} \sigma^{0}$ and $A_{1}=\cos \delta \operatorname{Im} \eta^{0}+\sin \delta \operatorname{Im} \sigma^{0}$. Their masses should be naturally around $v_{\Phi}$ to $v_{S}$. However, the fine-tuned case that light $H_{1}$ or $A_{1}$ is around few $\mathrm{GeV}$ cannot be ruled out. Which $Z_{\nu 2}$-odd degree of freedom is the viable dark matter candidate will be made clear soon.

Nonzero neutrino mass implies that the lepton flavor is no longer conserved. The null result in searching for one such process sets a stringent upper limit $\operatorname{Br}(\mu \rightarrow e \gamma)<1.2 \times 10^{-11}$ [1]. The contribution from active neutrinos is highly suppressed by the Glashow-IlipoulosMaiani mechanism so the process is dominated by the new physics. The $\mu \rightarrow e \gamma$ like transition is attributed to a loop-generated dimension-six operator $\bar{L} \Phi \sigma^{\mu \nu} e_{R} F_{\mu \nu}$. And the $\mu \rightarrow e \gamma$ branching ratio ( normalized to $\mu \rightarrow e \bar{\nu}_{e} \nu_{\mu}$ ) can be estimated to be

$$
\sim\left(\frac{e\left|g_{\mu k}^{*} g_{e k}\right| v_{\Phi}}{\left(16 \pi^{2}\right) G_{F} \Lambda^{3}}\right)^{2} \sim 10^{-8} \times|g|^{4} \times\left(\frac{1 \mathrm{TeV}}{\Lambda}\right)^{6}
$$

where $g^{2}$ represent a general Yukawa coupling product in the one-loop diagram. The $\mu \rightarrow e \gamma$ process does not impose further constraint because in our scenario $|g| \sim 10^{-4}$ is set by the neutrino masses. The new physics impact on the neutrinoless double beta decay and $a_{\mu}$ is insignificant due to the residual $Z_{\nu 2}$ parity.

The gauge boson $Z_{\nu}^{\prime}$ can couple to the SM sector through a kinetic mixing term $-\frac{\epsilon}{2} B^{\mu \nu} X_{\mu \nu}$, where $B^{\mu \nu}\left(X^{\mu \nu}\right)$ is the SM hypercharge $\left(U(1)_{\nu}\right)$ field strength. The analysis of [11] works perfectly for this model. By taking $\epsilon \sim 0.07$, which makes the global electroweak precision tests fit worsen by 1\%, it was shown that the Drell-Yan production of a $\mathrm{TeV}$ range $Z_{\nu}^{\prime}$ at $\mathrm{LHC}$ is possible. Moreover, the TeV range $Z_{\nu}^{\prime}$ has definite relative decay branching ratios into the SM fermions which are determined completely by the hypercharge of fermion and the mixing parameter $\epsilon[11]: B\left(Z_{\nu}^{\prime} \rightarrow u \bar{u}\right): B\left(Z_{\nu}^{\prime} \rightarrow d \bar{d}\right): B\left(Z_{\nu}^{\prime} \rightarrow e \bar{e}\right):$ $B\left(Z_{\nu}^{\prime} \rightarrow \nu \bar{\nu}\right)=5.63: 1.66: 4.99: 1$. However, in the case that $\chi_{1}$ and $H_{1}, A_{1}$ are much lighter than $Z_{\nu}^{\prime}, Z_{\nu}^{\prime} \rightarrow \chi_{1} \chi_{1}, H_{1} H_{1}, A_{1} A_{1}$ will become the dominant decay channels. 


\section{COSMOLOGICAL CONSEQUENCES}

In this model, $\chi_{1}, H_{1}$, and $A_{1}$ are the potential cold dark matter candidates. If $\chi_{1}$ is the lightest $Z_{2 \nu}$-odd state, it annihilates into the SM leptons through the tree-level t- and u-channel diagrams mediated by $\eta$, see Fig.2. The annihilation cross section is given by (ignoring the SM lepton masses )

$$
\sigma_{a n n} v_{r e l}=\frac{v_{r e l}^{2}}{24 \pi M_{\chi}^{2}} \sum_{i j}\left|g_{i 1} g_{j 1}^{*}\right|^{2} x^{2}\left(1-2 x+2 x^{2}\right),
$$

where $x=M_{\chi}^{2} /\left(M_{\eta}^{2}+M_{\chi}^{2}\right)$ and $i, j$ stand for the final state lepton. However, given that the Yukawa coupling $\sim 10^{-4}, M_{\eta} \ll M_{\chi}$ is required [12] to yield the relic density of $\Omega_{\chi_{1}} h^{2} \sim 0.11$ [5], which contradicts the assumption that $\chi_{1}$ is the dark matter candidate. Since that
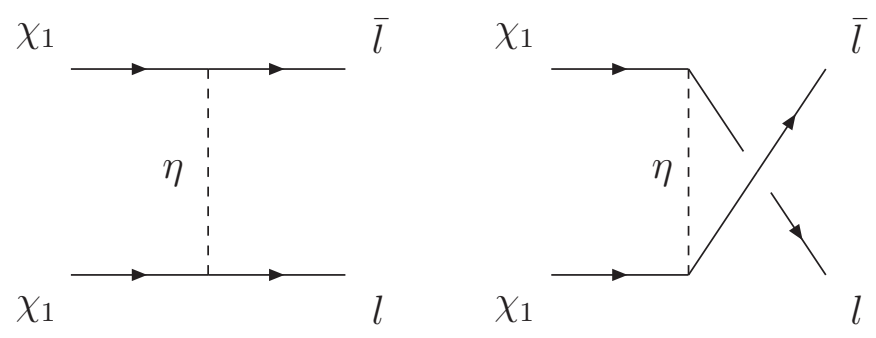

FIG. 2: The leading contribution(shown in the interaction basis) to the annihilation of the lightest $Z_{\nu 2}$-odd Majorana fermion $\chi_{1}$.

$M_{\chi_{1-4}}>M_{\eta}$, now we have to check that the densities of the four Majorana states diminish quickly as the universe cools down.

In the interaction basis, the decays of $\chi$ 's happen via the Yukawa coupling $g_{i a} \bar{L}_{i} \tilde{\eta} N_{a}+$ h.c. and the mixing between the $n_{L}$ and $N_{R}$ sectors, see Fig. The final state SM lepton can be

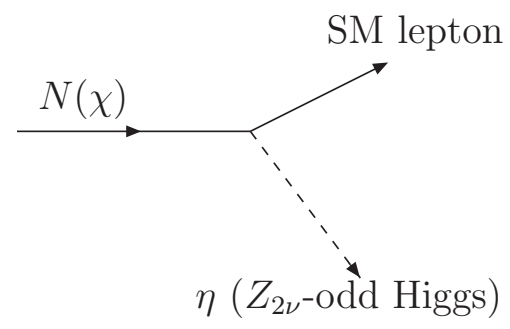

FIG. 3: The Feynman diagram in the interaction basis for $N$ to decay. The mass states are indicated in the parentheses. 
treated massless and the decay width can be carried out straightforwardly:

$$
\Gamma_{\chi}=\frac{|g|^{2}}{16 \pi} \times \sin ^{2} \theta \times M_{\chi}\left(1-\frac{m_{\eta}^{2}}{M_{\chi}^{2}}\right)^{2}
$$

where $\sin \theta$ represents all the mixing between the mass eigenstates and interaction eigenstates, also the inexplicit summation over all possible kinematically allowed channels is understood. Due to the working assumption that $M_{D} \sim y v_{S}$ the mixing between $\chi_{1-4}$ and $N$ are about order one, i.e. $\sin ^{2} \theta \sim \mathcal{O}(1)$, as discussed in Sec III. The decay rate is to be compared with the Hubble constant when temperature is around $\sim M_{\chi}$

$$
H\left(M_{\chi}\right)=\sqrt{\frac{4 \pi^{3} g_{*}}{45}} \frac{M_{\chi}^{2}}{M_{\text {Planck }}},
$$

where $g_{*} \sim 100$ is the effective number of degrees of freedom at temperature $\sim M_{\chi}$. With $|g|^{2} \sim 10^{-8}, m_{\eta} / M_{\chi} \sim \mathcal{O}(1)$, we find

$$
\frac{\Gamma_{\chi}}{H\left(M_{\chi}\right)} \sim\left(3.7 \times 10^{5}\right) \cdot \sin ^{2} \theta \cdot\left(1-\frac{m_{\eta}^{2}}{M_{\chi}^{2}}\right)^{2} \cdot\left(\frac{1 \mathrm{TeV}}{M_{\chi}}\right) \gg 1 .
$$

We conclude that the Majorana states decay into the SM final states and parity odd scalars fast enough as the Universe expands.

Therefore, either $H_{1}$ or $A_{1}$ is the viable dark matter candidate and all the heavier $Z_{2 \nu}$-odd scalars decay into the SM $W^{ \pm} / Z^{0}$ plus $H_{1}$ or $A_{1}$. We use $M_{S}$ to denote the lighter one of $M_{H_{1}}$ and $M_{A_{1}}$. For $M_{S}<m_{h^{0}}$, the leading order contribution to the scalar dark matter annihilation is through the SM Higgs exchange, see Fig $4(a)$. The annihilation cross section is given by [13]

$$
\sigma_{a n n} v_{r e l}=\frac{8 \lambda^{2} v_{\Phi}^{2} \sum_{i} \Gamma\left(h^{0} \rightarrow X_{i}\right)}{\left(4 M_{S}^{2}-m_{h^{0}}^{2}\right)^{2}+\Gamma_{h^{0}}^{2} m_{h^{0}}^{2}} \frac{1}{2 M_{S}}
$$

where $\lambda=\cos ^{2} \alpha\left(\lambda_{5}+\lambda_{6}\right)+\sin ^{2} \alpha \lambda_{7}+\sin 2 \alpha\left(\mu_{2}+\kappa v_{S}\right) / v_{\Phi}$ for $H_{1}$, and $\lambda=\cos ^{2} \delta\left(\lambda_{5}+\right.$ $\left.\lambda_{6}\right)+\sin ^{2} \delta \lambda_{7}+\sin 2 \delta\left(\mu_{2}-\kappa v_{S}\right) / v_{\Phi}$ for $A_{1}$. Here $\Gamma_{h^{0}}$ is the total SM Higgs decay width, and $\Gamma\left(h^{0} \rightarrow X_{i}\right)$ is the partial rate for the virtual Higgs with mass around $2 M_{S}$ which decays into $X_{i}$. In the limit that $M_{S} \gg m_{h^{0}}$, the dominant final states are $W^{+} W^{-}, Z Z$ and $h^{0} h^{0}$ and the annihilation cross section becomes $\sigma_{\text {ann }} v_{r e l} \sim \lambda^{2} /\left(4 \pi M_{S}^{2}\right)$. When the masses of $H_{1}$ and $A_{1}$ are not too different from each other, one needs to include the coannihilation process, see Fig 4(b). A great deal of effort has been devoted to different aspects of the scalar dark matter including the relic density, its production and detection at the colliders, and its direct detection at various underground laboratories, see [13 15]. In short, all the 


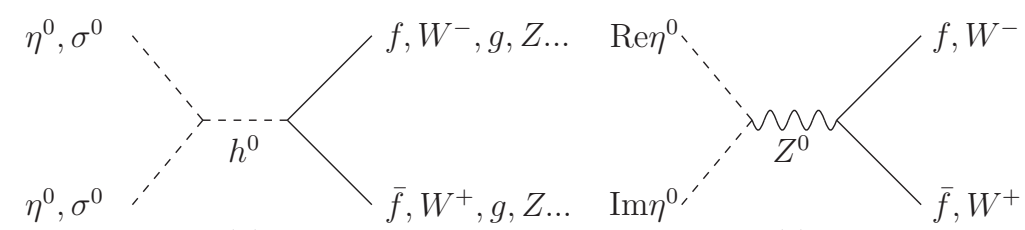

(a)

$(b)$

FIG. 4: The leading contribution (shown in the interaction basis) to the annihilation and coannihilation of the lightest $Z_{\nu 2}$-odd scalar.

studies agree that the scalar dark matter is viable and could be directly detected at the underground laboratories in the near future. However, to have the right dark matter relic density, $M_{S}$ and $\lambda$ are strongly correlated and such tight relation does not naturally come out in the general scalar dark matter models, and neither does it in this model.

The usual leptogenesis mechanism does not work in this model. The Yukawa coupling in our model is too large such that out of equilibrium condition cannot be met, see Eq.(13). To utilize the $\mathrm{TeV}$ scale singlet fermions for leptogenesis requires extra arrangement such as

the resonance leptogenesis[16] or via the three body decay mechanism[17]. But, some degree of fine-tuning is then unavoidable. We note in passing that the $Z_{2 \nu}$-odd scalar sector still helps to get a stronger first order electroweak phase transition which is crucial for successful electroweak baryogenesis.

\section{CONCLUSION AND DISCUSSION}

We construct a simple $U(1)_{\nu}$ gauge model to address the active neutrino mass generation, the testable new degrees of freedom beyond the SM, and the cold dark matter candidate at the same time. The active neutrino masses arise from one-loop diagrams, the effect is equivalent to a dim-7 operator at low energy, without much fine-tuning. The cold dark matter candidate is protected from decaying by a $Z_{\nu 2}$ parity a la KWM without extra global discrete or continuous symmetry introduced. The thermal relic density of the lightest $Z_{\nu 2^{-}}$ odd scalar can explain the observed dark matter abundance, albeit fine-tuning is required. All new fermions' masses are related to the SSB of $U(1)_{\nu}$ thus they can be probed at or below $\mathrm{TeV}$ scale. The lightest scalar and pseudoscalar can be pair produced associated with the SM Higgs through the $h^{0} H_{1} H_{1}, h^{0} A_{1} A_{1}$ vertices, or $H_{1} A_{1}$ can be produced via the $Z^{0} H_{1} A_{1}$ coupling. For the charged Higgs, it can be produced at the LHC via $p p \rightarrow W^{ \pm *} \rightarrow$ 
$H_{1} H^{ \pm}, A_{1} H^{ \pm}$or $p p \rightarrow \gamma^{*} / Z^{0 *} \rightarrow H^{\mp} H^{ \pm}$. The lightest Majorana fermion $\chi_{1}$ is most likely to be studied via the $U(1)_{\nu}$ gauge boson decay. Finally, the mass and mixing pattern in the lepton sector is not explained in this model. It could be purely accidental, due to some

flavor symmetry[18], or via the geometrical construction in higher dimensional scenarios[19].

\section{Acknowledgments}

Work was supported by the Taiwan NSC under Grant No. 99-2112-M-007-006-MY3.

[1] K. Nakamura et al. [Particle Data Group], J. Phys. G 37, 075021 (2010).

[2] T. Yanagida, in Proc. of the Workshop on Unified Theories and Baryon Number in the Universe (KEK, Tsukuba, 1979), edited by O. Sawada and A. Sugamoto, p. 95; M. Gell-Mann, P. Ramond, and R. Slansky, in Supergravity, edited by P. van Nieuwenhuizen and D. Z. Freedman (North-Holland, Amsterdam, 1979), p. 315; R. N. Mohapatra and G. Senjanovic, Phys. Rev. Lett. 44, 912 (1980); P. Minkowski, Phys. Lett. 67B, 421 (1977).

[3] A. Zee, Phys. Lett. B161, 141 (1985); A. Zee, Nucl. Phys. B264, 99 (1986); K. S. Babu, Phys. Lett. B203, 132 (1988).

[4] M. C. Chen and J. Huang, Mod. Phys. Lett. A 26, 1147 (2011) arXiv:1105.3188 [hep-ph]].

[5] M. Tegmark et al. [SDSS Collaboration], Phys. Rev. D 69, 103501 (2004); E. Komatsu et al. [WMAP Collaboration], Astrophys. J. Suppl. 192, 18 (2011).

[6] L. M. Krauss, S. Nasri and M. Trodden, Phys. Rev. D 67, 085002 (2003); K. Cheung and O. Seto, Phys. Rev. D 69, 113009 (2004); T. Asaka, S. Blanchet and M. Shaposhnikov, Phys. Lett. B 631, 151 (2005); E. Ma, Phys. Rev. D 73, 077301 (2006); T. Hambye, K. Kannike, E. Ma and M. Raidal, Phys. Rev. D 75, 095003 (2007); C. Boehm, Y. Farzan, T. Hambye, S. Palomares-Ruiz and S. Pascoli, Phys. Rev. D 77, 043516 (2008); M. Aoki, S. Kanemura and O. Seto, Phys. Rev. Lett. 102, 051805 (2009); Y. Farzan, Phys. Rev. D 80, 073009 (2009); Y. Farzan, S. Pascoli and M. A. Schmidt, JHEP 1010, 111 (2010); S. Kanemura, O. Seto and T. Shimomura, arXiv:1101.5713 [hep-ph].

[7] J. Kubo and D. Suematsu, Phys. Lett. B 643, 336 (2006).

[8] L. M. Krauss and F. Wilczek, Phys. Rev. Lett. 62, 1221 (1989). 
[9] B. Batell, Phys. Rev. D 83, 035006 (2011).

[10] see for example, A. E. Nelson, arXiv:1010.3970 [hep-ph].

[11] W. F. Chang, J. N. Ng and J. M. S. Wu, Phys. Rev. D 74, 095005 (2006) [Erratum-ibid. D 79, $039902(2009)]$.

[12] K. Griest, Phys. Rev. D 38, 2357 (1988) [Erratum-ibid. D 39, 3802 (1989)] [Phys. Rev. D 39, 3802 (1989)]. K. Griest, M. Kamionkowski and M. S. Turner, Phys. Rev. D 41, 3565 (1990). J. Kubo, E. Ma and D. Suematsu, Phys. Lett. B 642, 18 (2006).

[13] C. P. Burgess, M. Pospelov and T. ter Veldhuis, Nucl. Phys. B 619, 709 (2001).

[14] J. McDonald, Phys. Rev. D 50, 3637 (1994).

[15] H. Davoudiasl, R. Kitano, T. Li and H. Murayama, Phys. Lett. B 609, 117 (2005); R. Barbieri, L. J. Hall and V. S. Rychkov, Phys. Rev. D 74, 015007 (2006); Q. H. Cao, E. Ma and G. Rajasekaran, Phys. Rev. D 76, 095011 (2007); C. E. Yaguna, JCAP 0903, 003 (2009); M. Aoki, S. Kanemura and O. Seto, Phys. Rev. D 80, 033007 (2009); X. G. He, T. Li, X. Q. Li, J. Tandean and H. C. Tsai, Phys. Lett. B 688, 332 (2010); M. Farina, D. Pappadopulo and A. Strumia, Phys. Lett. B 688, 329 (2010); M. Aoki, S. Kanemura and O. Seto, Phys. Lett. B 685, 313 (2010); S. Kanemura, S. Matsumoto, T. Nabeshima and N. Okada, Phys. Rev. D 82, 055026 (2010); W. L. Guo and Y. L. Wu, JHEP 1010, 083 (2010); W. L. Guo and Y. L. Wu, arXiv:1103.5606 [hep-ph].

[16] A. Pilaftsis and T. E. J. Underwood, Nucl. Phys. B 692, 303 (2004).

[17] T. Hambye, Nucl. Phys. B 633, 171 (2002).

[18] see for example, H. Ishimori, T. Kobayashi, H. Ohki, Y. Shimizu, H. Okada and M. Tanimoto, Prog. Theor. Phys. Suppl. 183, 1 (2010), and the references therein.

[19] W. F. Chang, J. N. Ng and J. M. S. Wu, Phys. Rev. D 80, 113013 (2009); W. F. Chang, I. T. Chen and S. C. Liou, Phys. Rev. D 83, 025017 (2011). 